\title{
Educación superior a distancia. Una modalidad educativa posibilitadora de inclusión
}

\author{
Manzanilla-Granados, Héctor Manuel \\ Escuela Superior de Cómputo del Instituto Politécnico Nacional, Ciudad de México. \\ hmanzanilla@ipn.mx
}

\begin{abstract}
Navarrete-Cazales, Zaira
Facultad de Filosofía y Letras-Universidad Nacional Autónoma de México, Ciudad de México. znavarretec@gmail.com
\end{abstract}

\section{Resumen}

La educación superior a distancia (también conocida como «en línea», «virtual» o «e-Learning») en México es una posibilidad para quienes no pu eden tener acceso a la educación en las modalidades presencial y semipresencial. Esta modalidad de educación está ofertándose de manera acelerara en las universidades públicas como una manera de atender a la población vulnerable, que no tiene la posibilidad de acceso a la universidad convencional, con ello las universidades aumentan su cobertura a la cada vez mayor demanda de educción publica (gratuita). Con estas acciones, las universidades públicas mexicanas han aumentado su matrícula y con ello la cobertura nacional en educación superior.

En este texto presentaremos un análisis comparativo sobre: a) el crecimiento de la oferta de educación superior a distancia en dos los últimos dos sexenios presidenciales, y b) sobre los retos que enfrenta esta modalidad educativa para ser más incluyente. Una de las conclusiones es que a la población estudiantil en esta modalidad educativa, se les demanda la capacidad de autorregulación y habilidades comunicativas así como de pensamiento lógico; lo cual en muchas de las ocasiones no poseen debido a que estas poblaciones -las vulnerables- se han escolarizado en opciones educativas "pobres» y el contexto social en el que se desenvuelven no ha posibilitado el desarrollo de estas habilidades, lo cual lleva a que muchos estudiantes deserten de sus estudios en los primeros periodos escolares o sus bajos resultados. Así, el panorama de la educación a distancia es complejo y debe ser atendido con cuidado por las instituciones al momento de fijar su atención en una población vulnerable o en rezago educativo.

\section{Abstract}

The higher distance education (also known as "on-line", "virtual" or "e-learning") in Mexico is a possibility for those who can not have access to education in face - to - face and semipresencial modalities. This modality of education is being offered in an accelerated manner in public universities as a way to serve the vulnerable population, which does not have the possibility of access to the conventional university, with this the universities increase their coverage to the growing demand for public education (free). With these actions, mexican public universities have increased their enrollment and with it the national coverage in higher education.

In this text, we will present a comparative analysis on: a) the growth of the offer of higher distance education in the last two presidential six-year terms, and $b$ ) on the challenges that this educational modality faces to be more inclusive. One of the conclusions is that the student population in this educational modality, they are asked for the capacity of self-regulation and communication skills as well as for logical thinking; which in many of the occasions they do not have because these populations -the vulnerable- have been schooled in "poor" educational options and the social context in which they develop has not made possible the development of these skills, which leads many students to drop out of their studies in the first school periods or their low results. Thereby, the panorama of distance education is complex and must be taken care of by institutions when setting their attention to a vulnerable population or educational backwardness.

Palabras clave: Educación superior, modalidad a distancia, e-Learning, políticas, inclusión.

Keywords: higher education, distance learning, e-Learning, policies, inclusión.

\section{INTRODUCCIÓN}

El 25 de septiembre de 2015, la Asamblea General de la ONU adoptó la Agenda 2030 para el Desarrollo Sostenible, un plan de acción a favor de las personas, el planeta y la prosperidad, que también tiene la 
intención de fortalecer la paz universal y el acceso a la justicia. Los Estados miembros de la Naciones Unidas aprobaron una resolución en la que reconocen que el mayor desafío del mundo actual es la erradicación de la pobreza y afirman que sin lograrla no puede haber desarrollo sostenible (ONU, 2015).

La Agenda plantea 17 Objetivos con 169 metas de carácter integrado e indivisible que abarcan las esferas económica, social y ambiental. El Objetivo 4. Educación de calidad. Supone garantizar una educación inclusiva, equitativa y de calidad y promover oportunidades de aprendizaje durante toda la vida para todos. La educación es la base para mejorar nuestra vida y el desarrollo sostenible. Por tanto, el acceso a una educación de calidad ha de ser universal. En los últimos quinquenios, se han producido importantes avances con relación a la mejora de su acceso a todos los niveles y con el aumento en las tasas de escolarización, sobre todo, en el caso de las mujeres y las niñas. También se ha mejorado en gran medida el nivel mínimo de alfabetización (ONU, 2015).

No obstante, es necesario aumentar los esfuerzos para lograr mayores avances en materia de cobertura en la educación para todos, en cada uno de los distintos niveles de educación, consideramos que la educación a distancia posibilitará ampliar la cobertura de educación superior, en tal sentido, en este texto, presentamos un breve análisis comparativo sobre: a) el crecimiento de la oferta de educación superior a distancia en dos los últimos dos sexenios presidenciales en México, y b) sobre los retos que enfrenta esta modalidad educativa para ser más incluyente.

\section{CRECIMIENTO DE LA OFERTA DE EDUCACIÓN SUPERIOR A DISTANCIA EN MÉXICO}

De acuerdo con Amador (2010), en las últimas dos décadas del siglo XX, el Estado mexicano se transformó de un «Estado de bienestar» en un «Estado neoliberal» orientado por las políticas de los organismos mundiales. En este sentido, las políticas gubernamentales se enfocaron a la reestructuración de la educación superior como factor de desarrollo económico, mediante estrategias y acciones de planeación y racionalización del financiamiento de las universidades públicas, el crecimiento de las universidades privadas y la inversión en TIC. El reordenamiento y la evaluación del sistema de educación superior se plantearon como prioridades nacionales, con el propósito adaptarse a las nuevas exigencias del mercado mundial.

Las principales tendencias de la educación superior en América Latina, y por ende en México, se pueden resumir en: expansión cuantitativa, crecimiento de la privatización, diversificación institucional, restricción del gasto público y diáspora de talentos (UNESCO, 1995). Estas tendencias traen asociados varios problemas, entre los cuales se encuentran: inversión pública reducida; cambios en las políticas de los gobiernos; burocratización; rigidez de las estructuras y relaciones con la empresa y la producción. Ante esta situación son varios los retos que la educación superior en México debe enfrentar. Entre estos últimos se pueden mencionar: la actualización permanente de las competencias de los profesores y de los contenidos educativos; la introducción de redes electrónicas para el aprendizaje; la traducción y la adecuación de los principales aportes de las ciencias; la modificación de la gestión de las instituciones en un sentido menos jerárquico y formal; las políticas públicas ad hoc y propiciar el mutuo enriquecimiento entre distintas modalidades de educación (pública-privada) y entre diferentes instituciones educativas (formal, informal, no formal) (Castellanos y Luna, 2009).

Ante tal panorama, se hizo patente la necesidad de ofertar educación a distancia como una forma de inclusión para los sectores más pobres que no podían tener acceso a la educación presencial. La educación a distancia es una de las alternativas educativas más interesantes y potenciales tanto para los adultos que tienen dificultades de acceso a la educación como para los estudiantes regulares de las universidades (Parra, 2008). La educación a distancia implica la aplicación de nuevas tecnologías al proceso educativo; la formación y actualización docente para los sistemas abiertos. En esta tesitura, la UNESCO, (2008) planteó que para vivir, aprender y trabajar con éxito en una sociedad cada vez más compleja, rica en información y basada en el conocimiento, los estudiantes y los docentes debían utilizar la tecnología digital con eficacia. En un contexto educativo sólido, las TIC pueden ayudar a los estudiantes a adquirir las capacidades necesarias para llegar a ser: competentes para utilizar tecnologías de la información; buscadores, analizadores y evaluadores de información; solucionadores de problemas y tomadores de decisiones; usuarios creativos y eficaces de herramientas de productividad; comunicadores, colaboradores, publicadores y productores; y ciudadanos informados, responsables y capaces de contribuir a la sociedad. 
La oferta de educación a distancia por parte de universidades públicas, pero sobre todo privadas, creció de manera rápida en los años noventa y de manera exponencial durante la primera década de siglo XXI. Tales fueron los casos de la implementación de la educación a distancia en casi todas las universidades públicas del país. Conviene señalar que en México, existen 716 Universidades Públicas (9 Universidades Públicas Federales; 34 Universidades Públicas Estatales; 23 Universidades Públicas Estatales con Apoyo Solidario; 132 Institutos Tecnológicos Federales; 104 Universidades Tecnológicas; 50 Universidades Politécnicas; 12 Universidades Interculturales; 6 Centros Públicos de Investigación; 261 Escuelas Normales Públicas y; 85 Otras instituciones públicas) (SEP, 2014). Actualmente, la gran mayoría de éstas ofertan educación a distancia y algunas de estas universidades han creado en su interior Universidades Virtuales. A continuación mencionaremos, a manera de ejemplo, tres casos en donde se implementó la educación a distancia (una universidad, un politécnico y la creación de una universidad abierta y a distancia): la Universidad Nacional Autónoma de México (UNAM), el Instituto Politécnico Nacional (IPN) y la Universidad Abierta y a Distancia de México (UnADM).

En la Universidad Nacional Autónoma de México en 1997, se reorganizó la Estructura Académica, y se estableció la Coordinación de Universidad Abierta y Educación a Distancia (CUAED) que tenía como propósito fundamental dos acciones: 1) reestructurar a la Coordinación para redefinir las funciones de las direcciones y la creación de su Consejo Asesor, así como revisar y actualizar el Estatuto del Sistema Universidad Abierta, los reglamentos y las normas aplicables, y 2) desarrollar la base tecnológica requerida, tanto en equipo como en programas, y desarrollar programas de formación y capacitación del personal de las entidades universitarias, con apoyo técnico, académico y de infraestructura por parte de la CUAED (UNAM, 2013). Si bien es cierto que, la UNAM da paso de manera formal e institucionalizada a la «educación a distancia» en 1997, también es cierto que desde la década del setenta esta universidad ya ofrecía educación abierta por medio del Sistema de Universidad Abierta ${ }^{1}$. Es decir, en los años setenta se le denominaba "educación abierta» pero ésta implica educación a distancia pues se debe dar un tratamiento al material impreso de tal suerte que el estudiante pueda realizar sus actividades sin la presencia del docente. Ahí ya está la educación a distancia aunque no se le nombre. Lo que sucedió en los noventa es que las tecnologías vinculadas al cómputo y al internet potencializaron de forma extraordinaria nuevas formas de educación a distancia que, incluso, pudiera decirse que desplazaron al significante educación abierta del mapa de las política educativas de finales del siglo XX.

Por otro lado, y de manera más reciente, el Instituto Politécnico Nacional, creó en el año 2007 el Polivirtual, aunque sus primeros pasos para consolidar un modelo educativo distinto al escolarizado datan de 1995. Es resultado de años de impulso a un proyecto de educación a distancia y mixta (asistencia a laboratorios y aulas), que aún enfrenta desafíos. El Polivirtual es una modalidad a distancia o mixta, basada en una plataforma educativa propia (Torres, 2012). Ofrece estudios de bachillerato, licenciatura, posgrado y servicios educativos complementarios en modalidades alternativas, innovadoras y flexibles con apoyo de las tecnologías de la información y las comunicaciones. Para garantizar la calidad de la atención a estudiantes y usuarios, en el Polivirtual confluyen los esfuerzos y recursos de distintas dependencias politécnicas, a saber: unidades académicas, áreas de coordinación académica, técnica y administrativa (IPN, 2013).

El año 2012, se anunció la aprobación de creación de la Universidad Abierta y a Distancia de México, como un órgano administrativo desconcentrado de la Secretaría de Educación Pública, con autonomía técnica, académica y de gestión (Art.1 DOF, 2012). En su artículo segundo se plantea que la Universidad tendrá por objeto prestar servicios educativos del tipo superior, en la modalidad no escolarizada, que será abierta y a distancia, mediante el uso de las TIC, respaldados en redes de conocimiento, tecnológicas y administrativas, cuyas características serán la flexibilidad, la calidad y la pertinencia y a su vez, deberá procurar, bajo principios de equidad y colaboración, la más amplia cobertura, a fin de que el mayor número de personas pueda cursar los estudios que imparta. La creación de la Universidad Abierta y a Distancia de México es un evento importante para la edu-

\footnotetext{
${ }^{1}$ En su origen, el Sistema Universidad Abierta se concibió como parte integral del proyecto de Reforma Universitaria impulsada por el doctor Pablo González Casanova, a inicios de la década de los setenta, como una opción educativa flexible e innovadora en sus metodologías de enseñanza y evaluación de los conocimientos, con criterios de calidad y normados por un Estatuto aprobado por el Consejo Universitario el 25 de febrero de 1972, y por un Reglamento aprobado el 2 de diciembre de 1997, ambos modificados el 27 de marzo de 2009 . Con este Sistema flexible, se propició el estudio independiente, y permitió que se eliminaran los obstáculos de horario, lugar, edad, trabajo, etcétera, que impedían que cualquier persona que cubriera los requisitos de ingreso pudiera optar por un título universitario (UNAM, 2013).
} 
cación superior en México, aunque desde hace algunos años, la educación abierta y a distancia ya formaba parte de la oferta educativa de algunas universidades mexicanas (DOF, 2012).

Con la implementación de la educación a distancia se intenta por un lado ampliar la cobertura en educación para alcanzar los objetivos dictados por los organismos internacionales, por otro se busca ser más inclusivos y formar en las TIC, tanto a los administrativos, como a los docentes y alumnos y con ello "estar a la vanguardia en educación» (por lo menos en cuanto a herramientas tecnológicas se refiere, aunque muchas veces algunas universidades del país no cuenten con la infraestructura necesaria para impartir educación a distancia).

\section{RETOS QUE ENFRENTA LA EDUCACIÓN SUPERIOR A DISTANCIA EN MÉXICO PARA SER MÁS INCLUYENTE}

La educación superior a distancia en México es una posibilidad real para quienes no pueden tener acceso a la educación en las modalidades presencial y semipresencial. La educación superior a distancia está ofertándose de manera acelerara en las universidades públicas como una manera de atender a la población vulnerable, que no tiene la posibilidad de acceso a la universidad convencional, con ello las universidades aumentan su cobertura a la cada vez mayor demanda de educción publica (gratuita). Con estas acciones las universidades mexicanas han aumentado su matrícula y con ello la cobertura nacional en educación superior. A la población estudiantil en esta modalidad educativa, se les demanda la capacidad de autorregulación y habilidades comunicativas así como de pensamiento lógico; lo cual en muchas de las ocasiones no poseen debido a que estas poblaciones -las vulnerables- se han escolarizado en opciones educativas «pobres» y el contexto social en el que se desenvuelven no ha posibilitado el desarrollo de estas habilidades, lo cual lleva a que muchos estudiantes deserten de sus estudios en los primeros periodos escolares o sus bajos resultados (Manzanilla, 2014).

De acuerdo con el Instituto Nacional de Estadística y Geografía (INEGI), en $201745.3 \%$ de la población mexicana cuenta con computadora (50,5 millones de personas), un $63.9 \%$ de los mexicanos son usuarios de la Internet (71,3 millones de personas) y un $72.2 \%$ son usuarios de telefonía móvil celular (80,7 millones de personas). Estas cifras reflejan la desigualdad socioeconómica aparejada con la desigualdad tecnológica entre los habitantes de nuestro país más acentuada aún, considerando la diferenciación entre la población urbana y rural. Dada esta condición, podemos ubicar entonces un amplísimo sector que no es usuario ni de computadora ni de conexión a la Internet. Incluso, de la población usuaria de la Internet, el $12.5 \%$ (13,8 millones de personas) lo hace sin tener una computadora propia. Por consiguiente, esta desigualdad tecnológica aumenta significativamente las desventajas sociales y educativas.

Desde hace poco más de dos décadas la Internet se ha convertido en una de las herramientas más importantes y útiles para apoyar los procesos de educación formal y capacitación de los mexicanos, es decir, del $53.3 \%$ de personas que reportaron los principales usos que realiza de la Internet, poco más de las dos terceras partes, esto es, 7 de cada 10 personas dentro del grupo de población de 6 años en adelante, la utiliza para fines educativos o de formación. Pero, por otro lado, este porcentaje también revela que en México aún falta por aprovechar más el potencial que tiene la Internet en los procesos de educación o capacitación de los mexicanos, ya que casi cuatro quintas partes de la población únicamente la utiliza con fines de acceder a contenidos audiovisuales, de entretenimiento y de acceso a redes sociales.

\section{CONCLUSIONES}

La educación a distancia supone la aplicación de nuevas tecnologías al proceso educativo. En el Foro Mundial sobre la Educación 2015, se estableció como uno de sus acuerdos promover oportunidades de aprendizaje de calidad a lo largo de la vida para todos, en todos los contextos y en todos los niveles educativos, así como a fortalecer la ciencia, la tecnología y la innovación. Se recomendó aprovechar las TIC para reforzar los sistemas educativos, la difusión de conocimientos, el acceso a la información, el aprendizaje efectivo y de calidad, y una prestación más eficaz de servicios (UNESCO, 2015). 
En México, el panorama de la educación a distancia es complejo y debe ser atendido con cuidado por las instituciones al momento de fijar su atención en una población vulnerable o en rezago educativo. Los cursos propedéuticos y el trabajo de tutoría son buenos ejemplos de cómo se puede remediar esta problemática, ya que la disponibilidad de infraestructura tecnológica para el empleo de las TIC, es que en 2016, de los 110 millones mexicanos, dentro del grupo de edad de 6 años en adelante, el $47.0 \%$ señala ser usuario de computadora, el $59.5 \%$ de Internet y el $73.6 \%$ de telefonía móvil celular. Entre estos existe un amplísimo sector que no es usuario de la computadora ni de Internet, lo cual es un indicador de que este sector de personas no solo no tienen acceso a estos recursos tecnológicos, sino que además carecen de alfabetización digital básica. Incluso, de los que son usuarios de Internet, el $12.5 \%$ (13,8 millones de personas) lo hace sin tener una computadora propia. Esta desigualdad tecnológica se traduce en una desventaja social y educativa.

Del grupo de personas que son usuarios de computadora e Internet, una proporción de ellos no dispone ni de computadora ni de conexión a Internet en su hogar. En un mundo globalizado y en el contexto de la sociedad de la información y del conocimiento, el acceso ciudadano a Internet debe constituirse en un derecho humano. La falta de recursos económicos es el factor principal por el cual los mexicanos que disponen de computadora no cuentan con conexión a la Internet. Esta condición pone a estas personas en desventaja social, tecnológica y educativa, lo cual es necesario revertir si anhelamos un país más inclusivo, equitativo y competitivo a nivel nacional e internacional.

\section{REFERENCIAS BIBLIOGRÁFICAS}

Amador, R. (2010). «La Educación Superior a Distancia en México. Realidades y tendencias» en Lupión y Rama (coords.) La Educación Superior a Distancia en América Latina y el Caribe. Realidades y tendencias. Brasil: UNISUL.

Castellanos, S. y Luna C. (2009). «La internacionalización y la globalización neoliberal en el contexto de la educación superior en México» en Revista Iberoamericana de educación, Organización de Estados Iberoamericanos para la Educación, la Ciencia y la Cultura (OEI), No. 49/7, pp. 1-10.

Diario Oficial de la Federación (DOF). (2012). «DECRETO que crea la Universidad Abierta y a Distancia de México». 19/01/2012. Recuperado de http://dof.gob.mx/nota_detalle.php?codigo=5230365\&fecha=19/01/2012.

Instituto Nacional de Geografía y Estadística (2017). «Encuesta Nacional sobre Disponibilidad y Uso de Tecnologías de la Información en los Hogares 2017». Recuperado de http://www.beta.inegi.org.mx/proyectos/enchogares/regulares/dutih/2017/.

IPN (2013). «Qué es polivirtual», en Instituto Politécnico Nacional. Recuperado de http://www.polivirtual.ipn.mx/que_es.html.

Manzanilla, H.M. (2014). «Tecnologías de la Información y la Comunicación: Una vía para la internacionalización del conocimiento», en Navarrete, Z, y Navarro, M.A. (Eds.), Internacionalización y Educación Superior, Estados Unidos de América: Palibrio/Sociedad Mexicana de Educación Comparada, pp. 276-297.

Parra, O. (2008). El estudiante adulto en la era digital. Revista Apertura. Año 8, No. 8. 35-50.

Secretaría de Educación Pública (SEP) (2014). Subsecretaría de Planeación y Evaluación de Políticas Educativas, Recuperado de http://www.spep.sep.gob.mx/index.php/component/content/article/221.

Torres, M. (2012). «Pervive estigma de que la educación abierta es de segunda: experta» en La jornada (28 de febrero de 2012).

UNAM, (2013). «¿Qué es el Sistema Universidad Abierta y Educación a Distancia (SUAyED)?» en Universidad Nacional Autónoma de México. Recuperado de http://distancia.cuaed.unam.mx/que_es.php.

UNESCO (1995) Documento de política para el cambio y el desarrollo en la educación superior. París: UNESCO. Recuperado de: http://unesdoc.unesco.org/images/0009/000989/098992s.pdf.

UNESCO (2008). Estándares de Competencia en TIC para docentes. Londres: UNESCO. Recuperado de http://www.eduteka.org/EstandaresDocentesUnesco.php. 
UNESCO (2015). World Education Forum 2015: «Equitable and inclusive quality education and lifelong learning for all by 2030. Transforming lives through education» Recuperado de: http://en.unesco.org/world-education-forum-2015/incheon-declaration.

Organización de las Naciones Unidas (ONU) (2015) La Asamblea General adopta la Agenda 2030 para el Desarrollo Sostenible. Recuperado de http://www.un.org/sustainabledevelopment/es/2015/09/la-asamblea-general-adopta-la-agenda-2030-para-el-desarrollo-sostenible/. 